\title{
Prevalence of impairing behavioral health problems in ED patients and association with ED utilization.
}

\author{
Setarah Mohammad Nader ${ }^{1}$, Paul Musey Jr., MD, MS, FACEP2 \\ ${ }^{1}$ Indiana University School of Medicine, ${ }^{2}$ Indiana University School of Medicine, \\ Department of Emergency Medicine.
}

\section{Background and Hypothesis:}

It has been observed that patients with poor mental health are relatively frequent users of the Emergency Departments (ED). The objective of this study is to evaluate the prevalence of numerous behavioral health domains (depression, anxiety, PTSD, substance abuse, and suicidality) in patients presenting to the Emergency Department and the association of each of these domains with ED utilization.

\section{Experimental Design or Project Methods:}

This prospective study seeks to enroll a convenience sample of 1000 Englishspeaking adults presenting to IU Health Methodist and Eskenazi Emergency Departments without psychiatric chief-complaints. Patients were assessed for behavioral health problems using the CAT-MH ${ }^{\mathrm{TM}}, \mathrm{PHQ}-8$ and GAD-7 screening tools, which were administered via tablet device. Additionally, data on disposition medical history, discharge diagnoses, and ED utilization in the 12 months before and after enrollment from electronic medical records and data from the Indiana Network for Patient Care (INPC) will be reviewed.

\section{Results:}

Over the course of five weeks, 375 patients have been enrolled. Of those $59.4 \%$ were female with an overall mean age of 46.1 (SD \pm 16.4 ); $52.9 \%$ were white and $39.8 \%$ black/African American. Among enrollees $42.2 \%$ screened positive for depression, $29.7 \%$ for anxiety, and $1.3 \%$ for suicidal ideation. Patients who screened positive for depression were predominately females ( $76.1 \%$ vs $23.9 \%)$, those who screened positive for anxiety were also predominately females $(71.6 \%$ vs. $28.4 \%)$. However, 3 out of the $5(60 \%)$ patients that screened positive for suicidal ideation were males. The preliminary analysis of GAD-7 showed of those enrolled 215 (57.5\%) had no anxiety, $157(42 \%)$ had mild-severe anxiety. PHQ-8 scores showed 194 (51.9\%) had no depression, $178(47.5 \%)$ had mild-severe depression. Similarly, CAT-MH results showed $216(57.8 \%)$ had no depression, 
$158(42.2 \%)$ had mild-severe depression, while 263 (70.3\%) had no anxiety and $111(29.7 \%)$ had mild-severe anxiety. Full data analysis including comparative analysis of the CAT-MH with PHQ-8 and GAD-7 scores will take place after 1000 patients have been enrolled and data has been received from the INPC.

\section{Conclusion and Potential Impact:}

In our sample, almost half of patients that visit the ED have screened positive for mental health problems. We believe that early identification and appropriate referral may reduce inappropriate ED utilization. 